\title{
Potencial fisiológico de sementes de brássicas com ênfase no teste de envelhecimento acelerado
}

\author{
Caroline J Costa ${ }^{1}$; Mário B Trzeciak ${ }^{2}$; Francisco A Villela ${ }^{2}$ \\ ${ }^{1}$ Embrapa Cerrados, C. postal 08223, 73310-970 Planaltina-DF; '²FAEM-UFPel, C. postal 354, 96010-900 Pelotas-RS; \\ caroline.costa@cpac.embrapa.br
}

\begin{abstract}
RESUMO
O presente trabalho comparou diferentes testes para a avaliação do potencial fisiológico de sementes de brássicas, com ênfase no teste de envelhecimento acelerado (TEA). Foram utilizados quatro lotes de sementes de repolho, cultivar Coração de Boi, quatro de sementes de couve-brócolis, cultivar Piracicaba Precoce, e cinco de sementes de couve, cultivar Georgia. Foram realizados os testes de germinação, primeira contagem de germinação, emergência de plântulas, condutividade elétrica e envelhecimento acelerado. Neste, foram empregados três procedimentos: tradicional (água), solu-

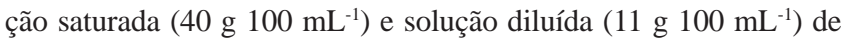
$\mathrm{NaCl}$, a $42^{\circ} \mathrm{C}$, por períodos de 48,72 e 96 horas. Foi utilizado o delineamento inteiramente casualizado, com quatro repetições e análises isoladas para cada teste. Os testes de primeira contagem de germinação e velocidade de emergência de plântulas apresentaram potencial para avaliação do potencial fisiológico de sementes de repolho e couve-brócolis. Já para sementes de couve, os testes de emergência de plântulas e condutividade elétrica foram os mais eficientes. TEA também foi eficiente na avaliação do potencial fisiológico de sementes de brássicas. Para repolho, a melhor distinção entre o potencial fisiológico das sementes através do TEA aconteceu seguindo o procedimento tradicional e empregando solução diluída de $\mathrm{NaCl}$, ambos por 48 e 72 horas, e solução saturada de $\mathrm{NaCl}$ por 72 e 96 horas. Para couve-brócolis, todas as variações do TEA testadas foram eficientes na identificação dos lotes de semente de qualidade superior. Já para couve, os melhores resultados foram obtidos aplicando TEA na metodologia tradicional por 96 horas e solução diluída por 72 horas. Observou-se ainda que, em comparação com o uso de água, a utilização de solução salina no TEA inibiu sensivelmente o crescimento e desenvolvimento de fungos.
\end{abstract}

Palavras-chave: Brassica oleracea var. capitata, B. oleracea var. italica, B. oleracea var. acephala, vigor, solução salina.

\begin{abstract}
Physiological potential of Brassica seeds with emphasis to the accelerated aging test
\end{abstract}

The present work compared different tests for the evaluation of the physiological potential of Brassica seeds with emphasis to the accelerated aging test (AAT). Four lots of cabbage seeds cv. Coração de Boi, four lots of broccoli seeds cv. Piracicaba Precoce and five lots of collard seeds cv. Georgia were used. Tests of germination, first germination counting, seedling emergence, electrical conductivity and accelerated aging were accomplished. In this test three procedures were used: traditional (water), saturated $\mathrm{NaCl}$ solution $(40 \mathrm{~g} / 100 \mathrm{~mL})$ and diluted $\mathrm{NaCl}$ solution $(11 \mathrm{~g} / 100 \mathrm{~mL})$, at $42^{\circ} \mathrm{C}$ for 48,72 and 96 hours. The experiment followed a completely randomized design with four replicates and individual evaluations for each test. The tests of first germination counting and speed of seedling emergence presented potential for evaluation of the physiological potential of cabbage and broccoli seeds. For collard seeds, the tests of seedling emergence and electrical conductivity were the most efficient. The AAT was also efficient in the evaluation of the physiological potential of Brassica seeds. For cabbage, the best distinction among the physiological potential of the seeds through the AAT was obtained following the traditional procedure and with diluted $\mathrm{NaCl}$ solution, both for 48 and 72 hours, and with saturated $\mathrm{NaCl}$ solution for 72 and 96 hours. For broccoli seeds, all of tested AAT procedures were efficient in the identification of the seed lots of superior quality. For collard seeds, the best results were obtained with the AAT in the traditional methodology for 96 hours and with diluted $\mathrm{NaCl}$ solution for 72 hours. It was observed that, in comparison to the use of water, the use of saline solution in the AAT inhibited sensibly the fungi growth and development.

Keywords: Brassica oleracea var. capitata, B. oleracea var. italica, B. oleracea var. acephala, vigour, saline solution.

\section{(Recebido para publicação em 25 de março de 2007; aceito em 9 de junho de 2008)}

\begin{abstract}
$\mathrm{A}$ procura por uma alimentação mais saudável e nutritiva tem levado a incrementos no consumo de hortaliças no país, estimulando o setor produtivo e aumentando a exigência quanto à qualidade dos produtos e processos empregados na condução dos campos de produção. No caso das brássicas, como repolho, couve-brócolis e couve, isso não é diferente, notadamente em razão de sua importância como alimentos funcionais.

Existem poucas informações referentes à avaliação do potencial fisiológico de sementes de brássicas. Consi-
\end{abstract}

derando que a utilização de sementes de alta qualidade é um pré-requisito para a obtenção de mudas vigorosas e uniformes, com reflexos no estande, produtividade e qualidade do produto colhido, é importante que existam informações que permitam avaliá-la. Sabe-se que a qualidade de um lote de sementes é representada pelo somatório de atributos genéticos, fisiológicos, sanitários e físicos que determinam o seu valor para semeadura, destacando-se o componente fisiológico da qualidade das sementes, diretamente responsável pelo seu desempenho em campo e no armazenamento (Rodo et al., 2000).

Vários testes têm sido empregados com o objetivo de estimar o desempenho das sementes em campo e/ou armazenamento, sendo o teste de envelhecimento acelerado um dos mais utilizados na avaliação do potencial fisiológico de sementes (Mello \& Tillmann, 1987; Vieira \& Carvalho, 1994; Ferguson-Spears, 1995). O teste baseiase no desempenho relativo entre lotes de sementes submetidos a condições de elevada temperatura e umidade relativa 
do ar, podendo-se estabelecer diferenças no potencial fisiológico das sementes conforme sua maior ou menor resistência à deterioração artificial provocada durante o teste (Marcos Filho, 1999). Desde o seu desenvolvimento e aplicação na avaliação do potencial fisiológico de sementes de hortaliças, o teste de envelhecimento acelerado tem proporcionado a obtenção de resultados menos precisos e reproduzíveis, relativamente às demais espécies. É provável que sementes que absorvam água mais rapidamente, como é o caso da maioria das sementes de hortaliças, atinjam teores de água muito variáveis ao final do teste de envelhecimento acelerado, prejudicando a uniformidade entre as amostras e a eficiência do teste na avaliação do potencial fisiológico das sementes. Estudos vêm sugerindo a substituição da água por soluções salinas saturadas ou diluídas, como forma de adequar a metodologia do teste de envelhecimento acelerado para sementes de hortaliças (Jianhua \& McDonald, 1996; Panobianco \& Marcos Filho, 1998, 2001; Rodo et al., 2000; Rodo \& Marcos Filho, 2003; Costa, 2004; Ávila, 2005). Dependendo da solução e da concentração utilizadas, são obtidos níveis específicos de umidade relativa do ar, possibilitando o ajuste da taxa de absorção de água, o que afeta a velocidade e intensidade de deterioração das sementes (Jianhua \& McDonald, 1996).

O presente trabalho teve como objetivo comparar a eficiência de diferentes testes para avaliação do potencial fisiológico de sementes de brássicas, com ênfase no teste de envelhecimento acelerado.

\section{MATERIAL E MÉTODOS}

O trabalho foi conduzido no Laboratório Didático de Análise de Sementes da Faculdade de Agronomia Eliseu Maciel, da Universidade Federal de Pelotas. Foram utilizados quatro lotes de sementes de repolho (Brassica oleracea L. var. capitata L.), cultivar Coração de Boi, quatro de sementes de couve-brócolis (B. oleracea L. var. italica PLENCK), cultivar Piracicaba Precoce e cinco de sementes de couve (B. oleracea L. var. acephala DC), cul- tivar Georgia. Os lotes de cada hortaliça foram submetidos aos testes: Germinação - foram empregadas doze subamostras de 50 sementes de cada lote, distribuídas sobre duas folhas de papel tipo germitest, umedecidas com água destilada em quantidade equivalente a 2,5 vezes a sua massa. Em seguida, as sementes foram cobertas com mais duas folhas de papel, confeccionandose os rolos, que foram levados ao germinador, regulado a $20^{\circ} \mathrm{C}$, na presença de luz constante. As avaliações foram realizadas aos cinco e dez dias, conforme as Regras para Análise de Sementes (Brasil, 1992). A primeira contagem do teste de germinação correspondeu à percentagem de plântulas normais, cinco dias após a instalação do teste; Emergência de plântulas - empregaram-se bandejas de poliestireno expandido de 200 células contendo substrato comercial (Plantmax $\left.{ }^{\circledR}\right)$ e uma semente por célula. Foram utilizadas 400 sementes de cada lote, subdivididas em oito subamostras de 50 sementes. Observouse diariamente o número de plântulas normais, até o décimo-quarto dia. Os resultados foram apresentados como percentagem e velocidade de emergência das plântulas, esta última expressa pelo índice de velocidade de emergência de plântulas (Nakagawa, 1999), calculado através da fórmula IVE $=\mathrm{E}_{1} / \mathrm{N}$ $+\mathrm{E}_{2} / \mathrm{N}_{2}+\ldots+\mathrm{E}_{\mathrm{n}} / \mathrm{N}_{\mathrm{n}}$, onde: IVE = Índice de Velocidade de Emergência; $\mathrm{E}_{1}, \mathrm{E}_{2}$, $\mathrm{E}_{\mathrm{n}}=$ número de plântulas normais observadas nas primeira, segunda e última contagens, respectivamente; $\mathrm{N}_{1}, \mathrm{~N}_{2}$, $\mathrm{N}_{\mathrm{n}}$ = número de dias da semeadura até $\mathrm{a}$ primeira, segunda e última contagens, respectivamente; Condutividade elétrica - doze subamostras de 50 sementes de cada lote foram pesadas, colocadas em copos plásticos contendo $25 \mathrm{~mL}$ de água deionizada e mantidas a $20^{\circ} \mathrm{C}$ durante 24 horas. Após esse período, determinou-se a condutividade elétrica da solução, utilizando um condutivímetro. Os resultados foram expressos em $\mu \mathrm{S} \mathrm{cm}^{-1} \mathrm{~g}^{-1} \mathrm{de}$ sementes; Envelhecimento acelerado - para o teste de envelhecimento acelerado, 600 sementes de cada lote foram distribuídas sobre telas de alumínio, fixadas no interior de caixas plásticas (tipo gerbox) contendo 40 mL de água destilada (procedimento tradicional), solução saturada
(40 g $\left.100 \mathrm{~mL}^{-1}\right)$ ou diluída (11 g 100 $\mathrm{mL}^{-1}$ ) de $\mathrm{NaCl}$. As caixas foram fechadas e mantidas em câmara regulada a $42^{\circ} \mathrm{C}$ por 48,72 e 96 horas. Após cada período, as sementes foram submetidas ao teste de germinação, conforme metodologia descrita anteriormente, calculando-se a percentagem de plântulas normais no quinto dia após a instalação do teste.

O teor de água das sementes de cada lote foi determinado antes e após a condução do teste de envelhecimento acelerado, seguindo as recomendações das Regras para Análise de Sementes (Brasil, 1992), empregando o método da estufa, a $105 \pm 3^{\circ} \mathrm{C}$, por 24 horas.

Foi utilizado o delineamento inteiramente casualizado, com quatro repetições, sendo as análises realizadas separadamente para cada teste. Os valores observados de germinação, percentagem de emergência de plântulas e envelhecimento acelerado foram transformados em arc sen e, as médias, comparadas pelo teste de Tukey $(\mathrm{p}<0,05)$. As análises estatísticas foram executadas através do Sistema de Análise Estatística para Microcomputadores - SANEST (Zonta \& Machado, 1986).

\section{RESULTADOS E DISCUSSÃO}

A percentagem de emergência de plântulas e o teste de condutividade elétrica não foram eficientes para distinguir os lotes de sementes de repolho quanto ao seu potencial fisiológico. Já a primeira contagem do teste de germinação e a velocidade de emergência de plântulas permitiram melhor classificação dos lotes quanto ao seu potencial fisiológico, não claramente evidenciadas pelo teste de germinação (Tabela 1). Estes testes indicaram o lote 1 como o de melhor desempenho e os lotes 2 e 4 apresentando desempenho intermediário. Foi possível estabelecer classificação semelhante baseada nos testes de envelhecimento acelerado, seguindo o procedimento tradicional por 48 e 72 horas e, modificado, empregando solução diluída de $\mathrm{NaCl}$ por 48 e 72 horas ou solução saturada de $\mathrm{NaCl}$ por 72 e 96 horas (Tabela 2). Vale destacar que a utilização de solução saturada de $\mathrm{NaCl}$ por 72 horas, além de separar os lotes quan- 
Tabela 1. Germinação, primeira contagem de germinação, percentagem de emergência de plântulas, índice de velocidade de emergência e condutividade elétrica de lotes de sementes de repolho, couve-brócolis e couve (germination, first germination counting, seedling emergence, emergence speed index and electrical conductivity of cabbage, broccoli and collard greens seed lots). Pelotas, UFPel, 2005.

\begin{tabular}{lcccccc}
\hline Hortaliça & Lotes & $\mathbf{G}^{\mathbf{1}}(\mathbf{\%})$ & $\begin{array}{c}\mathbf{1}^{\mathbf{a}} \text { Contagem } \\
(\%)\end{array}$ & $\mathbf{E}^{2}(\%)$ & IVE $^{3}$ & $\begin{array}{c}\mathbf{C E}^{4} \\
\left(\mu \mathbf{~ c m ~ g ~}^{-1}\right)\end{array}$ \\
\hline Repolho & 1 & $96 \mathrm{a}$ & $93 \mathrm{a}$ & $95 \mathrm{a}$ & $11,48 \mathrm{a}$ & $344,3 \mathrm{a}$ \\
& 2 & $93 \mathrm{ab}$ & $82 \mathrm{c}$ & $96 \mathrm{a}$ & $10,21 \mathrm{~b}$ & $379,3 \mathrm{a}$ \\
& 3 & $86 \mathrm{c}$ & $67 \mathrm{~d}$ & $93 \mathrm{a}$ & $9,66 \mathrm{c}$ & $368,3 \mathrm{a}$ \\
& 4 & $91 \mathrm{bc}$ & $86 \mathrm{~b}$ & $95 \mathrm{a}$ & $10,55 \mathrm{~b}$ & $358,0 \mathrm{a}$ \\
\hline CV(\%) & & 3,9 & 2,0 & 3,0 & 2,2 & 5,5 \\
\hline Couve-Brócolis & 5 & $94 \mathrm{a}$ & $88 \mathrm{a}$ & $94 \mathrm{a}$ & $11,35 \mathrm{a}$ & $365,8 \mathrm{c}$ \\
& 6 & $94 \mathrm{a}$ & $84 \mathrm{ab}$ & $93 \mathrm{a}$ & $10,97 \mathrm{ab}$ & $208,2 \mathrm{ab}$ \\
& 7 & $89 \mathrm{~b}$ & $79 \mathrm{~b}$ & $90 \mathrm{ab}$ & $10,58 \mathrm{~b}$ & $189,0 \mathrm{a}$ \\
& 8 & $86 \mathrm{~b}$ & $70 \mathrm{c}$ & $85 \mathrm{~b}$ & $9,44 \mathrm{c}$ & $240,2 \mathrm{~b}$ \\
\hline CV(\%) & & 2,3 & 3,9 & 4,4 & 2,6 & 7,5 \\
\hline Couve & 9 & $82 \mathrm{~b}$ & $79 \mathrm{~b}$ & $68 \mathrm{~b}$ & $3,32 \mathrm{a}$ & $236,9 \mathrm{~b}$ \\
& 10 & $85 \mathrm{~b}$ & $81 \mathrm{~b}$ & $62 \mathrm{~b}$ & $2,79 \mathrm{a}$ & $238,0 \mathrm{~b}$ \\
& 11 & $94 \mathrm{a}$ & $91 \mathrm{a}$ & $83 \mathrm{a}$ & $3,49 \mathrm{a}$ & $123,2 \mathrm{a}$ \\
& 12 & $67 \mathrm{~d}$ & $56 \mathrm{~d}$ & $54 \mathrm{c}$ & $3,22 \mathrm{a}$ & $453,4 \mathrm{c}$ \\
& 13 & $77 \mathrm{c}$ & $70 \mathrm{c}$ & $60 \mathrm{bc}$ & $3,14 \mathrm{a}$ & $450,5 \mathrm{c}$ \\
\hline CV (\%) & & 1,9 & 2,1 & 6,4 & 7,0 & 5,7 \\
\hline
\end{tabular}

Médias seguidas de mesma letra na coluna, em cada hortaliça, não diferem entre si pelo teste de Tukey, $\mathrm{p}<0,05$ (means followed by the same letter in the column, to each vegetable, do not differ from each other, Tukey's test, $\mathrm{p}<0,05)$. ${ }^{1} \mathrm{G}=$ germinação (germination); ${ }^{2} \mathrm{E}=$ emergência de plântulas (seedling emergence); ${ }^{3} / \mathrm{IVE}=$ índice de velocidade de emergência (emergence speed index); ${ }^{4} \mathrm{CE}=$ condutividade elétrica (electrical conductivity). to ao potencial fisiológico, não causou deterioração acentuada das sementes.

Para as sementes de couve-brócolis, os resultados de emergência e velocidade de emergência e germinação e primeira contagem de germinação foram similares e indicaram a superioridade do potencial fisiológico das sementes dos lotes 5 e 6 (Tabela 1). Mendonça et al. (2003) já haviam observado a eficiência do teste de primeira contagem e da percentagem e velocidade de emergência de plântulas em detectar diferenças no potencial fisiológico entre lotes de sementes de couve-brócolis. Assim, embora a redução na velocidade de germinação das sementes não esteja entre os primeiros eventos associados ao processo de deterioração, segundo Delouche \& Baskin (1973), o teste de primeira contagem de germinação apresentou sensibilidade em detectar pequenas diferenças no potencial fisiológico entre lotes de sementes de repolho e couve-brócolis, apresentando potencial de utilização como um teste de vigor para essas espécies.

Tabela 2. Germinação em porcentagem de lotes de sementes de repolho, couve-brócolis e couve após três períodos de exposição ao teste de envelhecimento acelerado (EA) (germination in percentage of cabbage, broccoli and collard greens seeds after three exposure periods of the accelerated aging test). Pelotas, UFPel, 2005.

\begin{tabular}{|c|c|c|c|c|c|c|c|c|c|c|}
\hline \multirow{2}{*}{ Hortaliça } & \multirow{2}{*}{ Lotes } & \multicolumn{3}{|c|}{$\mathrm{EA}^{1}$} & \multicolumn{3}{|c|}{$\mathrm{EA}^{2}$} & \multicolumn{3}{|c|}{$E^{3}$} \\
\hline & & $48 h$ & $72 \mathrm{~h}$ & $96 \mathrm{~h}$ & $48 \mathrm{~h}$ & $72 \mathrm{~h}$ & $96 \mathrm{~h}$ & $48 h$ & $72 \mathrm{~h}$ & $96 \mathrm{~h}$ \\
\hline \multirow{4}{*}{ Repolho } & 1 & $47 a$ & $28 a$ & $4 a$ & $42 a$ & $59 a$ & $1 \mathrm{a}$ & $82 a$ & $88 a$ & $74 a$ \\
\hline & 2 & $6 c$ & $1 \mathrm{c}$ & $3 a b$ & $32 b$ & $32 \mathrm{~b}$ & $0 \mathrm{a}$ & $46 \mathrm{c}$ & $65 \mathrm{~b}$ & $22 \mathrm{bc}$ \\
\hline & 3 & $2 d$ & $0 \mathrm{c}$ & $0 \mathrm{~b}$ & $13 c$ & $2 c$ & $0 \mathrm{a}$ & $48 \mathrm{c}$ & $55 c$ & $21 c$ \\
\hline & 4 & $21 \mathrm{~b}$ & $11 \mathrm{~b}$ & $4 a$ & $45 a$ & $38 \mathrm{~b}$ & $2 \mathrm{a}$ & $70 \mathrm{~b}$ & $68 \mathrm{~b}$ & $29 \mathrm{~b}$ \\
\hline CV (\%) & & 11,5 & 14,8 & 5,1 & 4,5 & 6,8 & 6,5 & 3,9 & 3,9 & 5,7 \\
\hline \multirow{4}{*}{ Couve- Brócolis } & 5 & $79 a$ & $66 a$ & $45 a$ & $79 a$ & $72 a$ & $25 a$ & $80 a$ & $86 a$ & $69 a$ \\
\hline & 6 & $78 a$ & $61 \mathrm{a}$ & $13 b$ & $67 \mathrm{~b}$ & $69 a$ & $30 \mathrm{a}$ & $82 \mathrm{a}$ & $84 \mathrm{a}$ & $67 a$ \\
\hline & 7 & $50 \mathrm{~b}$ & $49 \mathrm{~b}$ & $3 c$ & $65 \mathrm{~b}$ & $69 a$ & $5 b$ & $75 b$ & $78 \mathrm{~b}$ & $56 \mathrm{~b}$ \\
\hline & 8 & $34 \mathrm{c}$ & $15 \mathrm{c}$ & $10 \mathrm{~b}$ & $38 \mathrm{c}$ & $38 \mathrm{~b}$ & $0 \mathrm{c}$ & $50 \mathrm{c}$ & $53 c$ & $27 c$ \\
\hline CV (\%) & & 3,3 & 5,2 & 10,6 & 4,1 & 3,2 & 12,6 & 2,8 & 2,9 & 5,0 \\
\hline \multirow{5}{*}{ Couve } & 9 & $10 \mathrm{e}$ & $27 \mathrm{~b}$ & $13 c$ & $53 c$ & $40 \mathrm{c}$ & $21 c$ & $66 \mathrm{~b}$ & $59 d$ & $58 \mathrm{bc}$ \\
\hline & 10 & $28 \mathrm{c}$ & $9 d$ & $17 \mathrm{~b}$ & $63 \mathrm{~b}$ & $57 \mathrm{~b}$ & $38 \mathrm{~b}$ & $70 \mathrm{~b}$ & $77 \mathrm{~b}$ & $65 \mathrm{~b}$ \\
\hline & 11 & $79 a$ & $70 a$ & $27 a$ & $79 a$ & $78 \mathrm{a}$ & $43 \mathrm{~b}$ & $91 \mathrm{a}$ & $92 a$ & $86 a$ \\
\hline & 12 & $23 d$ & $12 \mathrm{~d}$ & $7 d$ & $44 \mathrm{c}$ & $30 \mathrm{~d}$ & $22 \mathrm{c}$ & $52 \mathrm{c}$ & $50 \mathrm{e}$ & $49 c$ \\
\hline & 13 & $40 \mathrm{~b}$ & $20 \mathrm{c}$ & $8 d$ & $50 \mathrm{c}$ & $33 d$ & $48 \mathrm{a}$ & $67 \mathrm{~b}$ & $69 c$ & $65 \mathrm{~b}$ \\
\hline CV (\%) & & 4,5 & 6,0 & 4,1 & 4,6 & 3,8 & 3,5 & 3,9 & 2,6 & 4,2 \\
\hline
\end{tabular}

Médias seguidas de mesma letra na coluna, em cada hortaliça, não diferem entre si pelo teste de Tukey, $\mathrm{p}<0,05$ (means followed by the same letter in the column, to each vegetable, do not differ from each other, Tukey's test, $\mathrm{p}<0,05)$. ${ }^{1}$ Teste de envelhecimento acelerado seguindo a metodologia tradicional, com água (traditional accelerated aging test, with water); ${ }^{2}$ Teste de envelhecimento acelerado utilizando solução diluída de $\mathrm{NaCl}$ (accelerated aging test with diluted $\mathrm{NaCl}$ solution); ${ }^{3}$ Teste de envelhecimento acelerado utilizando solução concentrada de $\mathrm{NaCl}$ (accelerated aging test with saturated $\mathrm{NaCl}$ solution). 
Tabela 3. Teor de água de lotes de sementes de repolho, couve-brócolis e couve após três períodos de exposição ao teste de envelhecimento acelerado (EA) (Water content of cabbage, broccoli and collard greens seeds after three exposure periods of the accelerated aging test). Pelotas, UFPel, 2005.

\begin{tabular}{|c|c|c|c|c|c|c|c|c|c|c|c|}
\hline \multirow{2}{*}{ Hortaliça } & \multirow{2}{*}{ Lotes } & \multirow{2}{*}{ Inicial } & \multicolumn{3}{|c|}{$\mathrm{EA}^{1}$} & \multicolumn{3}{|c|}{$\mathrm{EA}^{2}$} & \multicolumn{3}{|c|}{$\mathrm{EA}^{3}$} \\
\hline & & & $48 h$ & $72 \mathrm{~h}$ & $96 \mathrm{~h}$ & $48 \mathrm{~h}$ & $72 \mathrm{~h}$ & $96 \mathrm{~h}$ & $48 \mathrm{~h}$ & $72 \mathrm{~h}$ & $96 \mathrm{~h}$ \\
\hline \multirow{4}{*}{ Repolho } & 1 & 5,8 & 31,6 & 31,9 & 31,5 & 16,9 & 16,7 & 17,2 & 9,0 & 9,1 & 9,3 \\
\hline & 2 & 5,5 & 27,3 & 28,4 & 28,0 & 17,0 & 16,7 & 17,2 & 9,4 & 9,3 & 9,0 \\
\hline & 3 & 5,9 & 23,8 & 23,9 & 24,2 & 16,8 & 17,4 & 17,1 & 9,7 & 9,5 & 9,4 \\
\hline & 4 & 5,7 & 30,7 & 31,0 & 31,2 & 17,3 & 17,0 & 16,9 & 9,3 & 9,2 & 9,2 \\
\hline \multirow{4}{*}{ Couve- Brócolis } & 5 & 5,4 & 31,0 & 31,9 & 32,3 & 17,3 & 17,0 & 16,9 & 9,8 & 9,5 & 9,6 \\
\hline & 6 & 5,3 & 25,7 & 26,6 & 26,3 & 16,8 & 16,9 & 16,8 & 9,4 & 9,7 & 10,0 \\
\hline & 7 & 5,0 & 27,8 & 29,3 & 28,9 & 16,5 & 16,8 & 17,1 & 9,7 & 9,8 & 9,8 \\
\hline & 8 & 5,1 & 27,0 & 27,1 & 27,0 & 17,0 & 16,8 & 17,3 & 9,4 & 9,0 & 9,5 \\
\hline \multirow{5}{*}{ Couve } & 9 & 5,4 & 22,4 & 22,0 & 22,5 & 17,9 & 18,1 & 18,3 & 9,7 & 9,5 & 9,7 \\
\hline & 10 & 5,7 & 21,1 & 22,1 & 21,7 & 18,2 & 17,9 & 18,0 & 9,2 & 9,3 & 9,3 \\
\hline & 11 & 5,5 & 24,8 & 25,6 & 24,6 & 18,3 & 18,0 & 18,3 & 9,5 & 9,1 & 9,4 \\
\hline & 12 & 5,5 & 24,9 & 25,0 & 25,3 & 17,8 & 17,7 & 17,9 & 9,6 & 9,1 & 9,5 \\
\hline & 13 & 5,8 & 21,0 & 21,8 & 22,0 & 17,9 & 18,0 & 17,8 & 9,6 & 9,4 & 9,4 \\
\hline
\end{tabular}

${ }^{1}$ Teste de envelhecimento acelerado seguindo a metodologia tradicional, com água (traditional accelerated aging test, with water); ${ }^{2}$ Teste de envelhecimento acelerado utilizando solução diluída de $\mathrm{NaCl}$ (accelerated aging test with diluted $\mathrm{NaCl}$ solution); ${ }^{3} \mathrm{Teste}$ de envelhecimento acelerado utilizando solução concentrada de $\mathrm{NaCl}$ (accelerated aging test with saturated $\mathrm{NaCl}$ solution).

A mesma tendência observada nesses testes repetiu-se de forma geral no teste de envelhecimento acelerado, empregando o procedimento tradicional, com água, ou o procedimento modificado, com solução saturada de $\mathrm{NaCl}$, nos três períodos avaliados (Tabela 2). Esses resultados contrariam aqueles obtidos por Martins et al. (2002), que não consideraram o teste de envelhecimento acelerado, conduzido segundo procedimento tradicional por 48 e 72 horas, eficiente na classificação de lotes de sementes de couve-brócolis. Todavia, Mello et al. (1999) também observaram a eficiência do teste de envelhecimento acelerado, conduzido a $42^{\circ} \mathrm{C}$ por 48 horas, no estabelecimento de diferenças no potencial fisiológico entre lotes de sementes de couve-brócolis.

Para os lotes de sementes de couve, verificou-se que a percentagem de emergência de plântulas e os resultados do teste de condutividade elétrica permitiram classificar os lotes de forma semelhante aos resultados do teste de germinação: o lote 11 apresentou desempenho superior, os lotes 9 e 10, desempenho intermediário e, os lotes 12 e 13, baixo potencial fisiológico. A velocidade de emergência de plântulas, por outro lado, não possibilitou diferenciar os lotes (Tabela 1). Os testes de envelhecimento acelerado seguindo a metodologia tradicional por 96 horas e empregando solução diluída de $\mathrm{NaCl}$ por 72 horas também possibilitaram o ranqueamento dos lotes de modo semelhante, em três níveis de potencial fisiológico (Tabela 2).

Observou-se que as sementes submetidas ao teste de envelhecimento acelerado empregando o procedimento tradicional atingiram maior teor de água que aquelas submetidas ao procedimento modificado. Isso pode ser explicado pela maior umidade relativa do ar em contato com as sementes nesse procedimento (Tabela 3). Ao se empregar solução saturada de $\mathrm{NaCl}$, a umidade relativa do ar em contato com as sementes é de $76 \%$ e, ao se utilizar solução diluída (11 g $100 \mathrm{~mL}^{-1} \mathrm{de} \mathrm{NaCl}$ ), é de $94 \%$, de acordo com a equação de van't Hoff (Salisbury \& Ross, 1992). As sementes tendem a alcançar o equilíbrio higroscópico em teores de água mais elevados conforme se aumenta a umidade relativa do ar. De acordo com Marcos Filho (2005), o teor de água correspondente ao ponto de equilíbrio aumenta com a elevação da umidade relativa do ar e vice-versa. Assim, sementes em contato com ar apresentando $100 \%$ de umidade relativa apresentarão maior teor de água que sementes em contato com ar apresentando $94 \%$ e $76 \%$ de umidade relativa, respectivamente.
Como a umidade relativa do ar em contato com as sementes durante o teste de envelhecimento acelerado seguindo o procedimento modificado é inferior em relação ao procedimento tradicional, esperava-se que o período de condução do teste fosse aumentado, dada a menor intensidade de deterioração sofrida pelas sementes. Entretanto, no presente trabalho, isso só ocorreu ao se avaliar o potencial fisiológico de sementes de repolho, sendo possível classificar os lotes de sementes em níveis de vigor a partir de 48 horas utilizando o procedimento tradicional e, somente a partir de 72 horas, utilizando o procedimento modificado (Tabela 2).

No caso das sementes de couve-brócolis, o período de 48 horas do teste de envelhecimento acelerado possibilitou a obtenção de resultados semelhantes, independentemente do procedimento adotado, conforme anteriormente observado em sementes de melão (Torres, 2002) e rúcula (Ramos et al., 2004). Isso revela que os efeitos da temperatura elevada foram mais drásticos do que os provocados pela elevação da umidade relativa do ar. Como a utilização de solução salina em substituição à água nas mini-câmaras de envelhecimento acelerado afeta apenas a umidade relativa do ar em contato com as sementes, esse procedimento não prejudica as informa- 
ções fornecidas pelo teste de envelhecimento acelerado modificado.

O teste de envelhecimento acelerado seguindo o procedimento tradicional resultou em maiores variações entre o teor de água dos lotes. Variações acima de 5,0 pontos percentuais foram observadas entre o teor de água dos lotes de sementes de repolho e couve-brócolis após o teste de envelhecimento acelerado utilizando o procedimento tradicional (Tabela 3). Por outro lado, ao se empregar o procedimento modificado, essa variação não atingiu 1,0 ponto percentual. Isso evidencia maior uniformidade do teor de água das sementes ao se utilizar o procedimento modificado, o que já foi observado em trabalhos anteriores, com sementes de beterraba (Costa, 2004) e rabanete (Ávila, 2005). De acordo com Marcos Filho (1999), um dos principais indicadores da uniformidade das condições do teste de envelhecimento acelerado é o teor de água das sementes ao final do teste. Variações de $3 \%$ a $4 \%$ entre as amostras são toleráveis. Acima desses limites, são consideradas excessivas e determinam a necessidade de repetição do teste.

Ressalta-se que, mesmo considerando que a utilização do procedimento tradicional ou modificado do teste de envelhecimento acelerado tenha possibilitado a obtenção de resultados semelhantes quanto à classificação dos lotes de sementes em relação ao seu potencial fisiológico, o procedimento empregando solução salina apresenta uma vantagem em relação ao procedimento tradicional: o uso de soluções salinas em substituição à água inibe sensivelmente o crescimento e desenvolvimento de fungos, facilitando o manuseio das sementes e eliminando sua possível interferência na avaliação do potencial fisiológico das sementes.

Uma análise geral dos resultados obtidos permite constatar que, na avaliação do potencial fisiológico de se- mentes de brássicas, assim como para outras espécies, sementes de variedades e até mesmo de cultivares diferentes podem exigir condições específicas para a condução de testes. Atenção especial deve ser dada ao teste de envelhecimento acelerado, uma vez que o genótipo afeta a tolerância das sementes ao estresse imposto durante o teste.

\section{REFERÊNCIAS}

ÁVILA PV. 2005. Teste de envelhecimento acelerado na avaliação do potencial fisiológico de sementes de rabanete. Pelotas: UFPel - FAEM. 20p (Dissertação mestrado).

BRASIL. 1992. Ministério da Agricultura e da Reforma Agrária. Regras para análise de sementes. Brasília: SNDA/DNDV/CLAV. $365 \mathrm{p}$.

COSTA CJ. 2004. Envelhecimento acelerado e condicionamento osmótico em sementes de beterraba. Pelotas: UFPel - FAEM. 31p. (Dissertação mestrado).

DELOUCHE JC; BASKIN CC. 1973. Accelerated aging techniques for predicting the relative storability of seed lots. Seed Science and Technology 1: 427-452.

FERGUSON-SPEARS J. 1995. An introduction to seed vigour testing. In: VAN DE VENTER, HA (ed). Seed vigour testing seminar. Zürich: ISTA. p. 1-10.

HAMPTON JG; COOLBEAR P. 1990. Potential versus actual seed performance: can vigour testing provide an answer? Seed Science and Technology 18: 215-228.

JIANHUA Z; McDONALD MB. 1996. The saturated salt accelerated aging test for smallseeded crops. Seed Science and Technology 25: 123-131.

MARCOS FILHO J. 1999. Teste de envelhecimento acelerado. In: KRZYZANOWSKI FC; VIEIRA RD; FRANÇA-NETO JB (eds). Vigor de sementes: conceitos e testes. Londrina: ABRATES. p. 3.1-3.24.

MARCOS FILHO J. 2005. Relações água/ semente. In: MARCOS FILHO J (ed). Fisiologia de sementes de plantas cultivadas. Piracicaba: FEALQ. p. 169-196.

MARTINS CC; MARTINELLI-SENEME A; CASTRO MM; NAKAGAWA J; CAVARIANI C. 2002. Comparação entre métodos para a avaliação do vigor de lotes de sementes de couve-brócolos (Brassica oleracea L. var. italica PLENK). Revista Brasileira de Sementes 24: 96-101.
MELLO SC; SPINOLA MCM; MINAMI K. 1999. Métodos de avaliação da qualidade fisiológica de sementes de brócolos. Scientia Agricola 56: 1151-1155. Suplemento.

MELLO VDC; TILLMANN MAA. 1987. O teste de vigor em câmara de envelhecimento precoce. Revista Brasileira de Sementes 9: 93102.

MENDONÇA EAF; RAMOS NP; FESSEL SA. 2003. Adequação da metodologia do teste de deterioração controlada para sementes de brócolis (Brassica oleracea L. var. italica). Revista Brasileira de Sementes 25: 18-24.

NAKAGAWA J. 1999. Testes de vigor baseados no desempenho de plântulas. In: KRZYZANOWSKI FC; VIEIRA RD; FRANÇA-NETO JB (eds). Vigor de sementes: conceitos e testes. Londrina: ABRATES. p. 2.1-2.21.

PANOBIANCO M; MARCOS FILHO J. 1998. Comparação entre métodos para avaliação da qualidade fisiológica de sementes de pimentão. Revista Brasileira de Sementes 20: 306-310.

PANOBIANCO M; MARCOS FILHO J. 2001. Envelhecimento acelerado e deterioração controlada em sementes de tomate. Scientia Agricola 58: 525-531.

RAMOS NP; FLOR EPO; MENDONÇA EAF; MINAMI K. 2004. Envelhecimento acelerado em sementes de rúcula (Eruca sativa L.). Revista Brasileira de Sementes 26: 98-103.

RODO AB; MARCOS FILHO J. 2003. Accelerated aging and controlled deterioration for the determination of the physiological potential of onion seeds. Scientia Agricola 60: 465-469.

RODO AB; PANOBIANCO M; MARCOSFILHO J. 2000. Metodologia alternativa do teste de envelhecimento acelerado para sementes de cenoura. Scientia Agricola 57: 289-292.

SALISBURY FB; ROSS CW. 1992. Plant physiology. 4ed. Belmont: Wadsworth. 682p.

TORRES SB. 2002. Métodos para avaliação do potencial fisiológico de sementes de melão. Piracicaba: USP-ESALQ. 103p. (Tese doutorado).

VIEIRA RD; CARVALHO NM. 1994. Testes de vigor em sementes. Jaboticabal: FUNEP. 164p.

ZONTA EP; MACHADO AA. 1986. Sistema de análise estatística para microcomputadores SANEST. Pelotas: UFPel, Instituto de Física e Matemática. $150 \mathrm{p}$. 